\title{
ATLAS and CMS Virtual Visits: Bringing Cutting Edge Science into the Classroom and Beyond
}

Marzena Lapka ${ }^{1}$

The CMS Experiment, CERN

CERN, Geneva, Switzerland

E-mail: Marzena.Lapkalcern.ch

\section{ATLAS Virtual Visits Team:}

\section{Steven Goldfarb}

The ATLAS Experiment, University of Michigan

CERN, Geneva, Switzerland

E-mail: Steven.Goldfarbacern.ch

\section{Lucie Aguirre}

The ATLAS Experiment, CERN

CERN, Geneva, Switzerland

E-mail: Lucie.Aquirredcern.ch

\section{Ewan Hill}

The ATLAS Experiment, University of Victoria

Victoria, Canada

E-mail: Ewan.Chin.Hillacern.ch

\section{Claire Adam-Bourdarios}

The ATLAS Experiment, Laboratoire de l'Accelerateur Lineaire CERN, Geneva, Switzerland

E-mail: Claire.Bourdariosdcern.ch

\section{CMS Virtual Visits Team:}

\section{Angelos Alexopoulos}

The CMS Experiment, CERN

CERN, Geneva, Switzerland

E-mail: Angelos. Alexpoulos acern.ch

\section{Noemi Beni}

The CMS Experiment, ATOMKI, Hungarian Academy of Sciences CERN, Geneva, Switzerland

E-mail: Noemi.Beniacern.ch

${ }^{1}$ Speaker 


\title{
Michael Hoch
}

The CMS Experiment, Austrian Academy of Sciences

CERN, Geneva, Switzerland

E-mail: Michael.Hochleern.ch

\section{Achille Petrilli}

The CMS Experiment, CERN

CERN, Geneva, Switzerland

E-mail: Achille.Petrilliacern.ch

\section{Zoltan Szillasi}

The CMS Experiment, ATOMKI, Hungarian Academy of Sciences

CERN, Geneva, Switzerland

E-mail: Zoltan.Szillasiecern.ch

\begin{abstract}
Advances in information and communications technologies (ICTS) have given rise to innovative uses of web-based video tools for global communication, enhancing the impact of large research facilities, including their outreach and education programmes. As an example, the Virtual Visits programmes developed by the ATLAS and CMS collaborations at CERN, use videoconferencing to communicate with schools and remote events around the globe. The goal of these programmes is to enable the public, especially young people, to become engaged in and understand the field of particle physics through direct dialogue between ATLAS/CMS scientists and remote audiences.
\end{abstract}

ATLAS and CMS collaborations enhanced the Virtual Visits concept in different ways, but still with the same objective, which is to break down geographical barriers and allow more people to enter the world of science, physics and particle physics. This supports local education and outreach activities. Both collaborations have hosted Virtual Visits for thousands of people from each continent with participants connecting from locations such as Kathmandu, Rio de Janeiro, Ghana, Riyadh, and even the South Pole. Audiences were mainly made up of high-school students and their teachers, but also included policy makers and the general public.

This paper gives an overview of the educational, technical and organizational aspects of both programmes, with their unique added value. We also present feedback collected from participants, followed by recommendations for future development envisaged for creating sustainable tools.

The European Physical Society Conference on High Energy Physics

22-29 July 2015

Vienna, Austria 


\section{Introduction}

In addition to performing research, one of the main responsibilities of the High-Energy Physics (HEP) community is to inform society. Communications efforts from CERN and the major Large Hadron Collider (LHC) collaborations have resulted in the general public taking a greater interest in the LHC in recent years. The Higgs boson's discovery in July 2012 and the Nobel Prize for Physics announcement in October 2013 [1] drew worldwide attention to CERN, ATLAS [2] and CMS. [3]

This paper, discusses how recent advances in information and communications technologies (ICTs) have brought new communication possibilities for the largest of the LHC collaborations: ATLAS and CMS, each comprising over 3000 members from around the globe. We also show how outreach teams from both collaborations, together with educators, are embracing webbased video tools to provide out-of-the-classroom experiences through the Virtual Visits programme. This connects students and teachers, no matter where their location, with researchers at CERN. A whole new world of teaching and learning opportunities becomes available.

\section{Virtual Visits - expanding the boundaries of communication}

Virtual Visits are live conversations that connect the public from around the globe, usually teachers and their students with scientists at CERN. These connections are possible because of the increased availability of videoconferencing systems offering new opportunities and innovative practices that are emerging in classrooms. Virtual Visits allow the participants not only to get remote access to CERN's facilities, but also to learn how the ATLAS and CMS detectors were built and how major scientific collaborations operate them.

A pilot Virtual Visit at CERN took place on 6 October 2003 when 200 physics students from schools around Ireland were taken for a virtual tour by CERN physicists. [4] This premier gave a good basis for ATLAS and CMS Experiments to establish Virtual Visits [5] [6] as one of their education and outreach projects in the future with its primary aim of bringing science to students and the general public around the globe, and to inspire them to enter into a dialogue with researchers from both collaborations.

Each year, up to 45,000 people visit the ATLAS and CMS experimental sites in person. Virtual Visits extend this visit experience to thousands around the globe who are unable to visit the laboratory in person. This list comprises a very broad geographical spread from Australia to the USA Pacific West Coast, from South Africa to Scandinavia. The map below [figure 1] shows locations from where Virtual Visits connections have already taken place. 


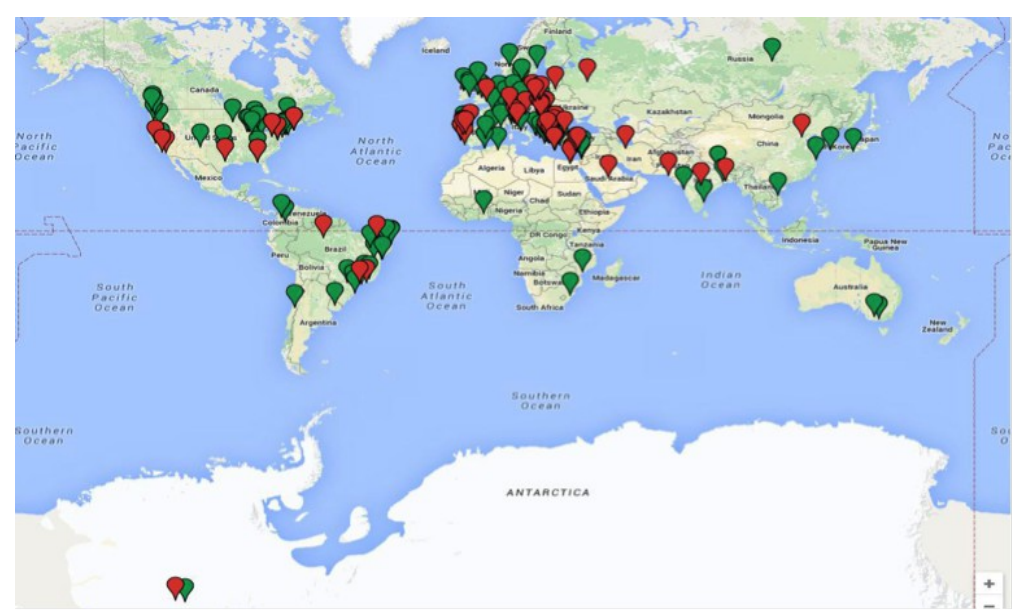

Figure 1: Map of the world showing ATLAS and CMS Virtual Visits connections. Green: ATLAS Virtual Visits; data from Nov 2010 - July 2015. Red: CMS Virtual Visits; data from Sept 2014 - July 2015.

Both teams observed that around $80 \%$ of the requests for Virtual Visits to ATLAS and CMS come from local contacts and events developed by the institutes that are part of the collaborations. Others were attracted by the collaborations' outreach programmes.

The most common example of a Virtual Visit is to a classroom of students, typically following the completion of an exercise such as an ATLAS or CMS Masterclass [7]. In addition to being included in the educational environment, virtual visits have also played an important role in events (such as science festivals, science cafés, Researchers' Nights) [8] and conferences, as can be seen on the diagram below [figure 2].

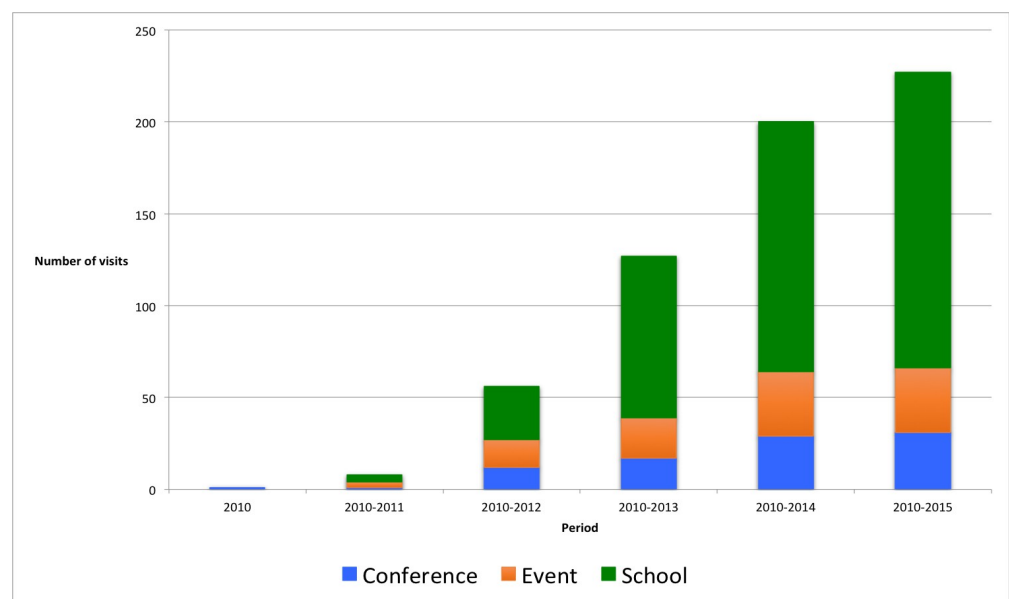

Figure 2: Cumulative diagram showing evolution of ATLAS Virtual Visits per type of audience.

\section{Technology employed}

Access to the CERN communication infrastructure allows ATLAS and CMS to reach worldwide audiences in an effective and cost-efficient manner. The connections are achieved by employing a combination of web-based video-conferencing [9], webcasting and audio-video hardware. Remote locations need a relatively straightforward set-up that consists of a computer, Internet, 
video projector/TV screen, microphone and a videoconferencing system that is simple to install and run. Additionally, other software such as Skype or Google Hangouts can be used. The latter was previously used for the "Hangout With CERN" series. [10]

There are some minor differences in the way both experiments handle the technical side of Virtual Visits. Detailed explanations of the technology employed for ATLAS Virtual Visits can be found in CHEP proceedings 2012 [11], while CMS Virtual Visits technical explanations are linked from CMS Communications Indico pages. [12]

\section{ATLAS Virtual Visits}

\subsection{History}

The ATLAS Virtual Visit project was initiated in Autumn, 2010. Audio and video equipment, already installed in the ATLAS Control Room for the public web casting of major events, such as LHC startup and first high-energy collisions, was integrated with a videoconferencing system. This allowed for live interaction between the public and members of the collaboration in the control room. It also provided the possibility to simultaneously webcast the event to passive participants and to record the event for future viewing.

Collaboration members tested the system by hosting visits typically from audiences either associated to their home institutes or from their home countries, making evident the value of the global and multilingual nature of the visits. An example of such a visit is this one from a high school in Greece [13]. All visits feature at least one host at the control room; many of them also feature a host located down by the detector or in one of the LHC facilities, to give the audience a "behind-the-scenes" view of the technology at CERN.

Over the past six years, the ATLAS Control Room has hosted 227 visits from 40 countries and all seven continents. The project was recognised as "Best Online Event" by the Digital Communication Awards [14] in 2013 and was a finalist for the European Excellence Award in 2014 [15].

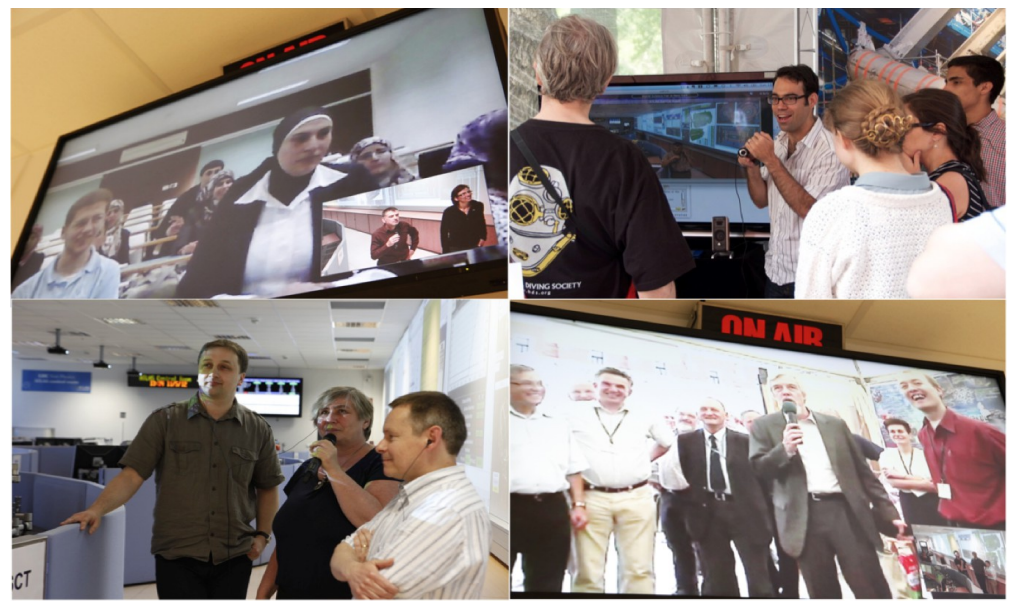

Figure 3: Clockwise from top-left: Visit from Birzeit University, Palestine; Visit from World Science Fair, New York City; CERN Director General. 


\subsection{Virtual Visits as Communication Training}

Initial testing of the project demonstrated the value of the concept, not only for improving the reach and quality of communication, but also in the training of collaboration members to engage in public outreach. Requests for Virtual Visits come from a variety of audience types, each with different nationalities, languages, age, and educational backgrounds. Fortunately, the large international collaborations are able to provide hosts with matching backgrounds, provided the members learn how to communicate to the audiences.

Collaboration members best matching the needs of a particular audience are located, and many volunteer to act as hosts. Training can either be through a shadow-shift during a preceding visit or by co-hosting the visit with one of the more experienced hosts. In contrast to the more formal training required for local guides, the process is very simple and attractive to young scientists. Participants are included on a growing list of guides, many of whom later volunteer for other activities in outreach or communication.

\section{CMS Virtual Visits}

\subsection{History}

The inaugural CMS Virtual Visit took place in autumn 2011 with the participation of highschool students from the Oveges School in Hungary. Due to a technical stop that made access to the experimental cavern possible, the students were able not only to see the CMS detector live but also to talk to Hungarian CMS scientists who were located next to it, 100 meters underground. Since then, a small but steadily increasing number of participants, predominantly school students, were offered the opportunity to visit virtually the CMS experiment. [16] A notable example includes an international-level Virtual Visit that connected high-school students from Greece and the US with both the CMS detector and the IceCube Neutrino observatory in Antarctica. [17] Building on this experience and subsequent technical and organizational improvements, Virtual Visits were officially launched in September 2014. [18] A year later, 73 Virtual Visits were organized with the participation of nearly 8,000 people across twenty-one countries. 


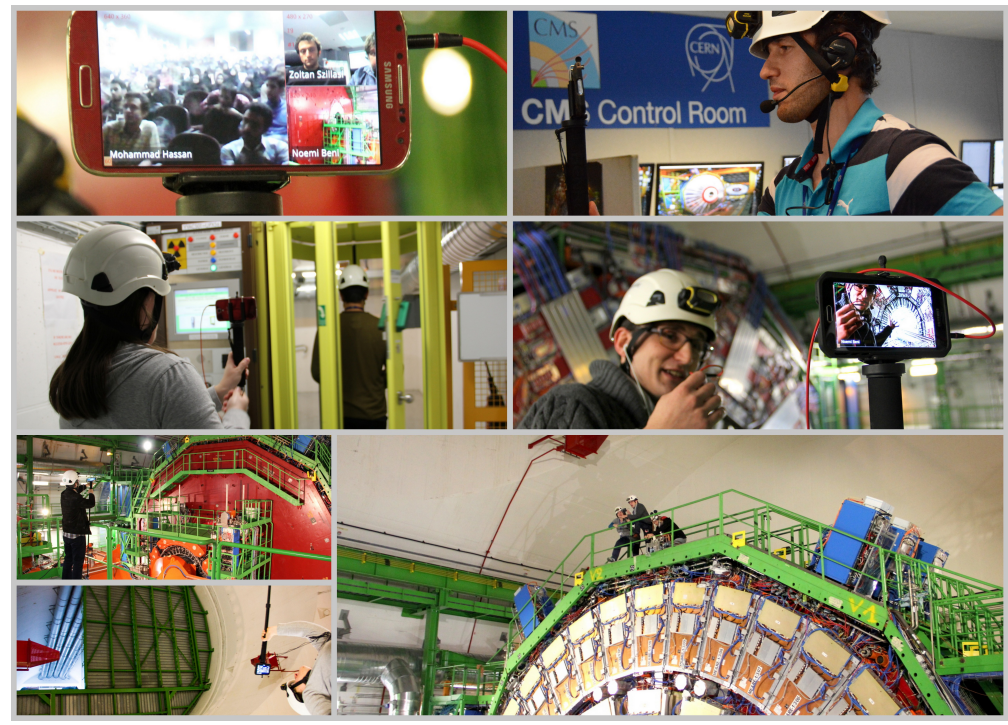

Figure 4: Clockwise from top-left: Mobile camera; CMS guide showing the Control Room; Passing through the iris scan to go 100 metres underground; Showing different angles of the CMS detector placed in its home cavern.

\subsection{Feedback on Virtual Visit}

Within a period of one year (September 2014 - September 2015) the CMS Virtual Visit team has collected quantitative and qualitative data from the local organizers and participants.

Quantitative indicators come from a post-visit survey filled by the local organizers. Twenty-five Virtual Visits, out of the 73 that took place in the mentioned period, were evaluated. Results reveal high levels of overall satisfaction with the CMS Virtual Visits among local organizers, with $96,6 \%$ of respondents confirming this in the survey. Furthermore, $98 \%$ of survey participants expect that the CMS Virtual Visit "will increase the interest of the participants in Science, Technology, Engineering and Mathematics (STEM)".

Qualitative feedback come from the reports, sent to the CMS Virtual Visits team after the visit, with the quotes from the participants. A few reflections presented below show that the main project goal to support local education and outreach was achieved, and there is an increase in the participants' interest in science after Virtual Visits.

"This way of gaining knowledge is way more attractive and interesting, because it lets us gather information directly from CERN workers on how one of the most important laboratories in Europe works, " said a 13-year-old student from Poland

"People here at NEDUET are now in action about particle physics, mission accomplished!" claimed CMS Virtual Visit participant from Pakistan

"What surprised me today is the fact that I didn't know the people were trying to find the answer about how the universe was made," affirmed an 11-year-old girl from Los Angeles (US) 


\section{Conclusions and Future Directions}

Today, the LHC enters another exciting phase with the second data-collection period at higher energies. Communication action is strategic to the success of the multi-decade research programme. Positive feedback and the steadily increasing number of requests supports the Virtual Visits' key goal of bringing the excitement of LHC research to students and the general public around the globe.

The Virtual Visits teams will continue to further develop and deliver a stable communication platform for their scientists in order to facilitate exchanges with society in their countries of origin. Given the desire to make sure virtual exchanges have an educational benefit, potential improvements can be made to assemble resources before and after the Virtual Visits experience. Also, by helping teachers prepare their students for the Virtual Visits experience in the best way possible, as well as maintaining their interest in Science, Technology, Engineering and Mathematics (STEM) afterwards.

Additional steps will also be taken on the organizational side, which is to train more guides and technical coordinators so that they can feel comfortable in their roles and build a stronger community that would support the growing demand.

\section{References}

[1] Higgs discovery (2012) and Nobel prize in physics (2013): http://home.web.cern.ch/topics/higgsboson

[2] The ATLAS Experiment on the Large Hadron Collider at CERN: $\underline{\text { http://atlas.ch/ }}$

[3] The CMS Experiment on the Large Hadron Collider at CERN: www.cern.ch/cms

[4] 200 Irish students at CERN - virtually! : https://cds.cern.ch/record/676868? $\ln =$ en

[5] ATLAS Virtual Visits website: $\underline{\text { http://cern.ch/atlas-virtual-visit }}$

[6] CMS Virtual Visits website: www.cern.ch/cms/content/virtual-visits

[7] LHC master classes in physics: http://physicsmasterclasses.org/index.php?cat=physics

[8] Researchers' Night: Science at the shops: http://home.web.cern.ch/about/updates/2015/10/researchers-night-science-shops

[9] Vidyo: IP-based video conferencing software: http://www.vidyo.com

[10] Hangout with CERN: Reaching the Public with the Collaborative Tools of Social Media, S. Goldfarb, K. Kahle, A. Rao, CHEP 2012, J. Phys.: Conf. Ser. $513 \underline{062019}$

[11] ATLAS Virtual Visits: Bringing the World into the ATLAS Control Room, S. Goldfarb, CHEP 2012, J. Phys.: Conf. Ser._396 $\underline{062008}$

[12] CMS Virtual Visits equipment is described here: https://indico.cern.ch/event/437672/contribution/1/attachments/1137562/1628337/VirtualVisitEquip ments_2015.pdf

[13] Recording of ATLAS Virtual Visit from Pelopio and Lala high schools in Greece: http://atlas-livevirtual-visit.web.cern.ch/atlas-live-virtual-visit/2012/Lala-2012.html 
[14] Digital Communication Awards, hosted by Quadriga University of Applied Sciences: http://www.digital-awards.eu (Award in 2013)

[15] The European Excellence Awards for leaders in Public Relations: http://www.excellence-awards.eu (Finalist in 2014)

[16] Hoch, A. \& Alexopoulos, A. (2014).ART@CMS and SCIENCE\&ART@SCHOOL: Novel Education and Communication Channels for Particle Physics. In Astroparticle, Particle, Space Physics and Detectors for Physics Applications-Proceedings of the 14th ICATPP Conference (Vol. 1, pp. 728-736)

[17] Example of CMS Virtual Visits with IceCube (joint ATLAS/CMS Virtual Visit): http://home.web.cern.ch/students-educators/updates/2014/01/cern-connects-icecube-bring-science$\underline{\text { schools }}$

[18] CMS open for Virtual Visits, from your school, science festival or exhibition centre: http://cylindricalonion.web.cern.ch/blog/201411/cms-open-virtual-visits-your-school-sciencefestival-or-exhibition-centre 